\title{
A viable compactification scenario in Gauss-Bonnet gravity
}

\author{
Alexey Toporensky ${ }^{1,2, \star}$ and Sergey Pavluchenko ${ }^{3, \star \star}$ \\ ${ }^{1}$ Sternberg Astronomical Institute, Moscow State University, Moscow 119991 Russia \\ ${ }^{2}$ Kazan Federal University, Kazan 420008 Russia \\ ${ }^{3}$ Programa de Pós-Graduação em Física, Universidade Federal do Maranhão (UFMA), 65085-580, São \\ Luís, Maranhão, Brazil
}

\begin{abstract}
In this talk we address two important issues which could affect reaching the exponential and Kasner asymptotes in Einstein-Gauss-Bonnet cosmologies - spatial curvature and anisotropy in both three- and extra-dimensional subspaces. In the first part we consider cosmological evolution of spaces being the product of two isotropic and spatially curved subspaces. We consider all possible number of spatial dimensions and provide description of the curvature effects in these dimensions. It is demonstrated that the dynamics in $D=2$ (the number of extra dimensions) and $D \geqslant 3$ is different. In particular, the regime with the "stabilization" of extra dimensions could be reached only if $D \geqslant 3$. In the second part we study the influence of initial anisotropy. Our study of reveals that transition from Gauss-Bonnet Kasner regime to anisotropic exponential expansion (with expanding three and contracting extra dimensions) is stable with respect to breaking the symmetry within both three- and extra-dimensional subspaces in any number of extra dimensions. This allows us to construct a scenario where isotropisation of outer and inner subspaces is reached dynamically from rather general anisotropic initial conditions.
\end{abstract}

In our study we deal with the cosmological dynamics in Lovelock gravity [1]. It has the following structure: its Lagrangian is constructed from terms

$$
L_{n}=\frac{1}{2^{n}} \delta_{j_{1} j_{2} \ldots j_{2 n}}^{i_{1} i_{2} \ldots i_{2 n}} R_{i_{1} i_{2}}^{j_{1} j_{2}} \ldots R_{i_{2 n-1} i_{2 n}}^{j_{2 n-1} j_{2 n}}
$$

where $\delta_{j_{1} j_{2} \ldots j_{2 n}}^{i_{1} i_{2} i_{2 n}}$ is the generalized Kronecker delta of the order $2 n$. One can verify that $L_{n}$ is Euler invariant in $D<2 n$ spatial dimensions and so it would not give nontrivial contribution into the equations of motion. So that the Lagrangian density for any given $D$ spatial dimensions is sum of all Lovelock invariants (1) upto $n=\left[\frac{D}{2}\right]$ which give nontrivial contributions into equations of motion (see e.g. [2]:

$$
\mathcal{L}=\sqrt{-g} \sum_{n} c_{n} L_{n}
$$

where $g$ is the determinant of metric tensor, $c_{n}$ is a coupling constant of the order of Planck length in $2 n$ dimensions and summation over all $n$ in consideration is assumed.

The most general spatially-flat metric Ansatz has the form

^e-mail: atopor@rambler.ru

$\star \star$ e-mail: sergey.pavluchenko@gmail.com 


$$
g_{\mu \nu}=\operatorname{diag}\left\{-1, a_{1}^{2}(t), a_{2}^{2}(t), \ldots, a_{n}^{2}(t)\right\}
$$

substituting it into the Lagrangian and varying with respect to the metric gives us the equations of motion:

$$
2\left[\sum_{j \neq i}\left(\dot{H}_{j}+H_{j}^{2}\right)+\sum_{\substack{\{k>l\} \\ \neq i}} H_{k} H_{l}\right]+8 \alpha\left[\sum_{j \neq i}\left(\dot{H}_{j}+H_{j}^{2}\right) \sum_{\substack{\{k>l\} \\ \neq\{i, j\}}} H_{k} H_{l}+3 \sum_{\substack{\{k>l>\\ m>n\} \neq i}} H_{k} H_{l} H_{m} H_{n}\right]-\Lambda=0
$$

as the $i$ th dynamical equation. The first Lovelock term - the Einstein-Hilbert contribution-is in the first set of brackets and the second term-Gauss-Bonnet-is in the second set; $\alpha$ is the coupling constant for the Gauss-Bonnet contribution and we put the corresponding constant for Einstein-Hilbert contribution to unity. Also, since we a consider spatially flat cosmological model, scale factors do not hold much in the physical sense and the equations are rewritten in terms of the Hubble parameters $H_{i}=\dot{a}_{i}(t) / a_{i}(t)$. Apart from the dynamical equations, we write down a constraint equation

$$
2 \sum_{i>j} H_{i} H_{j}+24 \alpha \sum_{i>j>k>l} H_{i} H_{j} H_{k} H_{l}=\Lambda .
$$

Prior to this study, we completed investigation of the most simple (but the most important as well) cases. The spatial part of these cases is the product of three- and extra-dimensional subspaces which are spatially flat and isotropic [3-5]. So that the obvious next step is consideration of these subspaces being non-flat and anisotropic, and that is what we have done in current investigation. Non-flatness is addressed by assuming that both subspaces have constant curvature while anisotropy - by breaking the symmetry between the spatial directions. The results of the curvature study suggest that the only viable regimes are those from the flat case with $\gamma_{(\mathbf{D})}=0$ requirement. Additionally, in the $\Lambda$-term case there is "geometric frustration" regime, described in [6,7] and further investigated in [8] with $\gamma_{(\mathbf{D})}=-1$ requirement.

First we investigate the impact of the spatial curvature on the cosmological regimes. As a "background" we use the results obtained in [3-5] - exact regimes for $\gamma_{(3)}=\gamma_{(\mathbf{D})} \equiv 0$ for both vacuum and $\Lambda$-term cases. As we use them as a "background" solutions, it is worth to quickly describe them all. All solutions found for both vacuum and $\Lambda$-term cases could be splitted into two groups - those with "standard" regimes as both past and future asymptotes and those with nonstandard singularity as one (or both) of the asymptotes. By the "standard" regimes we mean generalized Kasner solutions(when dependence of scale factor upon time is power-law) and exponential solutions (when scale factors depend exponentially on time, so that corresponding Hubble parameters are constant). In our study me encounter two different Kasner regimes - "classical" GR Kasner regime (with $\sum p_{i}=\sum p_{i}^{2}=1$ where $p_{i}$ is Kasner exponent from the definition of power-law behavior $\left.a_{i}(t)=t^{p_{i}}\right)$, which we denote as $K_{1}$ (as $\left.\sum p_{i}=1\right)$ and it is low-energy regime; and GB Kasner regime (with $\sum p_{i}=3$ ), which we denote as $K_{3}$ and it is high-energy regime. For realistic cosmology we should have high-energy regime as past asymptote and low-energy as future, but our investigation demonstrates that potentially both $K_{1}$ and $K_{3}$ could play a role as past and future asymptotes [3]. Also we should note that $K_{1}$ exist only in the vacuum regime, while $K_{3}$ as past asymptotes we encounter in both vacuum and $\Lambda$ term regimes (see [4] for details). The exponential regimes [9-11] could be seen in both vacuum and $\Lambda$-term regimes and there are two of them - isotropic and anisotropic ones. The former of them corresponds to the case where all the directions are isotropized and, since we work in the multidimensional case, it does not fit the observations. On contrary, the latter of them have different Hubble parameters for three- and extra-dimensional subspaces. For realistic compactification we demand expansion of 
the three- and contraction of the extra-dimensional spaces. The exponential solutions are denoted as $E_{i s o}$ for isotropic and $E_{3+D}$ for anisotropic, where $D$ is the number of extra dimensions (so that, say, in $D=2$ the anisotropic exponential solution is denoted as $E_{3+2}$ ).

The second large group are the regimes which have nonstandard singularity as either of the asymptotes or even both of them. The nonstandard singularity is the situation which arises in nonlinear theories and in our particular case it corresponds to the point of the evolution where $\dot{H}$ (the derivative of the Hubble parameter) diverges at the final $H$; we denote it as $n S$. Our previous research reveals that nonstandard singularity is a wide-spread phenomena in EGB cosmology, for instance, in $(4+1)$ dimensional Bianchi-I vacuum case all the trajectories have $n S$ as either past or future asymptote [12]. Since a nonstandard singularity means the beginning or end of dynamical evolution, either higher or lower values of $H$ do not reached and so the entire evolution from high to low energies cannot be restored; for this reason we disregard the trajectories with $n S$ in the present study.

So that the viable (or realistic) regimes are limited to $K_{3} \rightarrow K_{1}$ and $K_{3} \rightarrow E_{3+D}$ for vacuum case and $K_{3} \rightarrow E_{3+D}$ for $\Lambda$-term; these regimes we further investigate in the presence of curvature.

The results of the investigation suggest that all three considered cases have the original regime unchanged as long as $\gamma_{D}=0$. This means that the curvature of three-dimensional world alone cannot change the future asymptote. For nonzero curvature of the extra dimensions, the situation is different in all three cases: in vacuum $K_{3} \rightarrow E_{3+D}$ case all trajectories with $\gamma_{D} \neq 0$ are singular; in vacuum $K_{3} \rightarrow K_{1}$ we have two new regimes but both of them are nonviable; finally, in $\Lambda$-term $K_{3} \rightarrow E_{3+D}$ case if $\gamma_{D}>0$ the future asymptote is singular while for $\gamma_{D}<0$ there could be viable regime with stabilization of extra dimensions, but this regime occurs only when isotropic exponential solution cannot exist and in $D \geqslant 3$.

Our study also demonstrate the difference between $D=2$ (the lowest dimension where anisotropic exponential solution could exist [4]) and $D \geqslant 3$ case: in the former of them, the regime with stabilization cannot be reached while in the latter it is reachable.

To conclude, it seems that the only important player in this case is the curvature of extra dimensions. And this is clear why is it so - from requirements of viability we demand that three-dimensional subspace should expand while extra dimensions should contract. The expansion of the three dimensions cannot be stopped by neither curvature alone. On the other hand, extra dimensions are contracting, so both signs of extra-dimensional curvature affect it - positive usually leads to singularity (standard or not) while negative could turn it to expansion. The latter could even change the dynamics in three-dimensional sector, what we also see in $K_{3+D}^{i s o}$ regime.

The anisotropy was studied numerically - we found an exact $(3+D)$ anisotropic exponential solution (according to [3-5]) and "anizotropize" metric around it (consider totally anisotropic cosmology with $H_{1} \sim H_{2} \sim H_{3} \sim H$ and $H_{4} \sim \cdots \sim H_{D+3} \sim h$ where $H$ and $h$ are Hubble parameters for exact solution $E_{3+D}$ ). Then we fix all but two Hubble parameters and vary the remaining two to see how the evolution changes with variation of the initial conditions.

The results of anisotropy study reveal that the viable $K_{3} \rightarrow K_{1}$ (in vacuum) and $K_{3} \rightarrow E_{3+D}$ (in both vacuum and $\Lambda$-term) regimes are always stable with respect to breaking the isotropy in both subspaces, meaning that within some vicinity of exact transition, all initial conditions still lead to this regime (see Fig. 1). The difference is in the area of the basin of attraction - for $K_{3} \rightarrow K_{1}$ it is quite small (see Fig. 1(a)) while for $K_{3} \rightarrow E_{3+D}$ (the particular example is $K_{3} \rightarrow E_{3+2}$ ) it is much larger and unbounded (see Fig. 1(b)). As a way of example, we also provided the typical evolution curves for $K_{3} \rightarrow E_{3+D}$ in Fig. 2(a) and $K_{3} \rightarrow E_{\text {iso }}$ in Fig. 2(b).

Our study clearly demonstrates that the dynamics of the non-flat cosmologies could be different from flat cases and even some new regimes could emerge. Here we covered only the simplest case with constant-curvature subspaces leaving the most complicated cases aside - we are going to investigate some of them deeper in near future. 

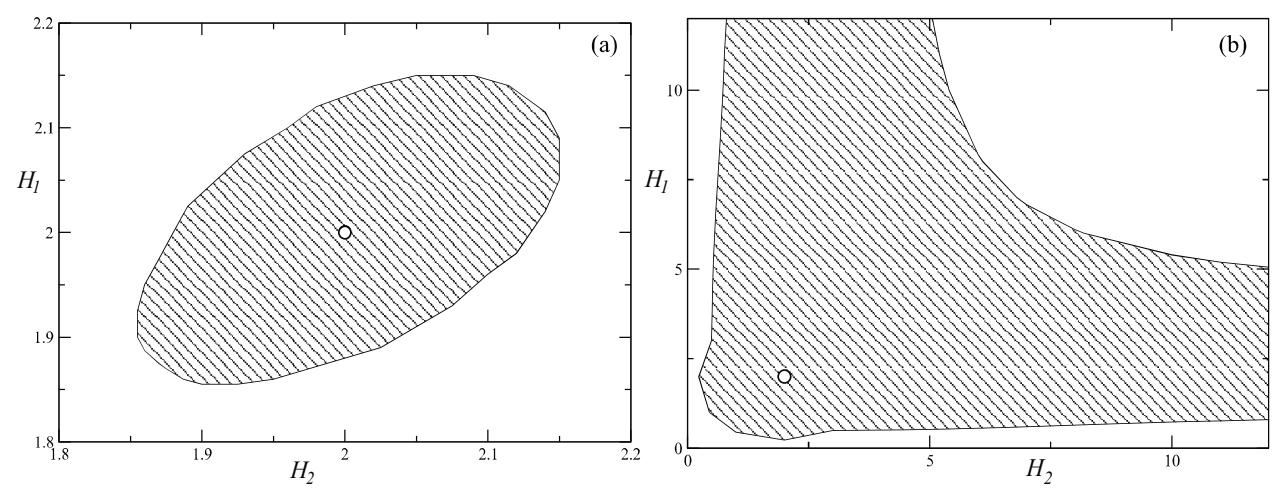

Figure 1. Typical stability areas for vacuum $K_{3} \rightarrow K_{1}$ regime on (a) panel; vacuum and $\Lambda$-term $K_{3} \rightarrow E_{3+D}$ regime on (b) panel (see the text for more details).

On the other hand, anisotropy of the subspaces can decrease the initial conditions leading to exact solution and through this diminish the chances for successful compactification. Our thorough investigation of totally anisotropic (Bianchi-I-type) five-dimensional EGB model [12] is a good example the only future asymptote in the vacuum case is nonstandard singularity. And since the isotropy (even within the subspace) could be treated as some sort of fine-tuning, independence of the final asymptote on the isotropy highly improve the chances for the model to be accepted, so that this direction of study is also important.

The previously described results allow us to construct a scenario of compactification which satisfy two important requirements:

- the evolution starts from a rather general anisotropic initial conditions,

- the evolution ends in a state with three isotropic big expanding dimensions and stabilized isotropic extra dimensions.

The first part of the scenario in question uses the results of anisotropy investigation. We have seen there that while starting from a quite wide area of the initial conditions, the flat anisotropic Universe tends to the exponential solution with three equal expanding dimensions.

Suppose also, that a negative spatial curvature is small enough at the beginning and starts to be important only after this transition to exponential solution (which is established in the present investigation only for a flat Universe) already occured. This condition allows us to glue the second part of the scenario which requires negative spatial curvature of the inner space. When describing the effects of curvature we saw that the exponential solution turn to the solution with stabilized extra dimensions in this case. As a result of these two stages a Universe starting from initially anisotropic both outer expanding three dimensional space and contracting extra dimensional inner space evolve naturally to the final stage with isotropic three big dimensions and isotropic and stabilized inner extra dimensional space. The only additional condition for this scenario to realize is that spatial curvature should become dynamically important only after the transition to exponential solution occurs.

This finalize our talk. The presented analysis suggests that more in-depth investigation of both curvature and anisotropy effects are required - we have investigated and described the most simple but still very important cases - constant-curvature and flat anisotropic (Bianchi-I-type) geometries; in the near future we are going to consider more complicated topologies. 


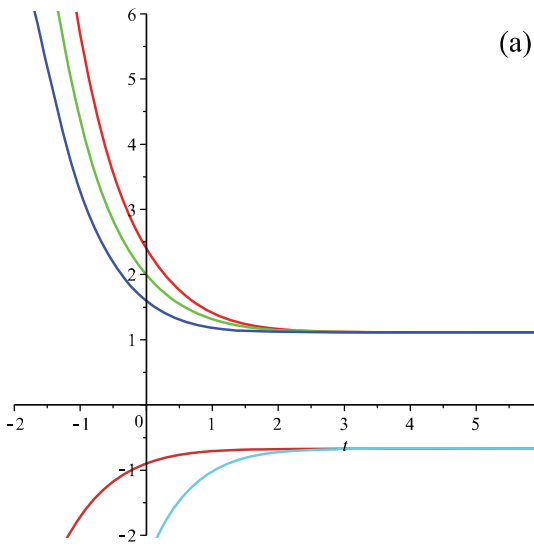

(a)

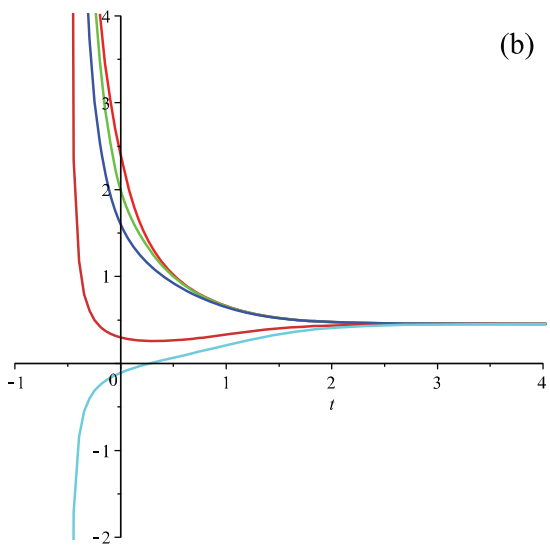

Figure 2. Typical evolution curve for stable anisotropic (a) and isotropic (b) exponential solutions with broken symmetry in both subspaces (see the text for more details).

Acknowledgments S.A.P. was supported by FAPEMA under project BPV-00038/16. The work of A.T. is supported by RFBR grant 17-02-01008 and by the Russian Government Program of Competitive Growth of Kazan Federal University. Authors are grateful to Alex Giacomini (ICFM-UACh, Valdivia, Chile) for discussions.

\section{References}

[1] D. Lovelock, J. Math. Phys. (N.Y.) 12, 498 (1971).

[2] S.A. Pavluchenko, Phys. Rev. D 80, 107501 (2009).

[3] S.A. Pavluchenko, Phys. Rev. D 94, 024046 (2016).

[4] S.A. Pavluchenko, Phys. Rev. D 94, 084019 (2016).

[5] S.A. Pavluchenko, Eur. Phys. J. C 77, 503 (2017).

[6] F. Canfora, A. Giacomini and S. A. Pavluchenko, Phys. Rev. D 88, 064044 (2013).

[7] F. Canfora, A. Giacomini and S. A. Pavluchenko, Gen. Rel. Grav. 46, 1805 (2014).

[8] F. Canfora, A. Giacomini, S. A. Pavluchenko and A. Toporensky, Friedmann dynamics recovered from compactified Einstein-Gauss-Bonnet cosmology, Grav. Cosmol. (accepted), arXiv: 1605.00041

[9] D. Chirkov, S. Pavluchenko, A. Toporensky, Mod. Phys. Lett. A29, 1450093 (2014).

[10] D. Chirkov, S. Pavluchenko, A. Toporensky, Gen. Rel. Grav. 46, 1799 (2014).

[11] D. Chirkov, S. Pavluchenko, A. Toporensky, Gen. Rel. Grav. 47, 137 (2015).

[12] S.A. Pavluchenko, Phys. Rev. D 82, 104021 (2010).

[13] S.A. Pavluchenko, Phys. Rev. D 92, 104017 (2015).

[14] V. D. Ivashchuk, Eur. Phys. J. C 76, 431 (2016). 